\title{
Fundoscopy screening for intraocular candida in patients with positive blood cultures-is it justified?
}

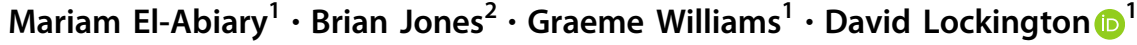

Received: 8 January 2018 / Revised: 24 May 2018 / Accepted: 30 May 2018 / Published online: 6 July 2018

(c) The Royal College of Ophthalmologists 2018

\begin{abstract}
Introduction Although the worldwide prevalence of disseminated candidaemia is rising, reported intraocular candidiasis rates are variable, even as low as $1 \%$. The Infectious Diseases Society of America recommends fundoscopy screening for all fungal blood culture positive patients. We wished to evaluate the impact of this recommendation on our department.

Methods A retrospective observational study was performed in NHS Greater Glasgow and Clyde (population $=1.15$ million) for all patients with candida positive blood culture results over a 2-year period.

Results From January 2015 to December 2016, 258 candida positive cultures were obtained from 168 adults (mean age $=$ 62 years, range: $17-94$ years; 85 females, 83 males). Candida species were isolated in 161/168 (95.8\%) cases $(43.5 \%$ Candida albicans, 35.7\% Candida glabrata). All 168 cases were treated with intravenous antifungals. 84 patients $(50 \%)$ were formally referred to ophthalmology. Of those not referred, 21 were deceased prior to culture result (12.5\%) and 14 patients subsequently deteriorated (8.3\%). Six patients reported visual symptoms. In total, $65 \%$ had no ocular findings and $32.5 \%$ had unrelated ocular signs. Only one patient had signs consistent with Candida chorioretinitis, making the prevalence of intraocular candida in our population $1.3 \%$ (1/80).

Conclusions The prevalence of ocular candidiasis is low, presumably due to potent systemic antifungal agents and good intraocular penetration. Our findings support the view that routine fundoscopy screening may not be indicated in every culture positive patient. This paper provides an evidence base for the Royal College's Eyecare in intensive care unit recommendations regarding targeted screening of non-verbal, symptomatic or high-risk patients.
\end{abstract}

\section{Introduction}

The worldwide prevalence of disseminated candidaemia is rising, possibly due to the growing population of immunocompromised patients [1]. A 2007 prospective survey reported prevalence rates of $4.8 / 100,000$ per population and 5.9/100,000 per acute hospital admissions in Scotland [2]. Risk factors for invasive candidiasis include: prolonged hospital stays, long intravascular lines, parenteral nutrition and recent abdominal surgery $[1,3,4]$.

David Lockington

davidlockington@hotmail.com

1 Tennent Institute of Ophthalmology, Gartnavel General Hospital, 1053 Great Western Road, Glasgow G12 0YN, UK

2 Institute of Infection, Immunity \& Inflammation, University of Glasgow, Glasgow, UK
Endogenous ocular involvement is a rare complication, but can be sight threatening. Historical reports of intraocular candidiasis have published prevalence rates approaching $28 \%$, but more recent publications suggest it can be as low as $1 \%[1,5,6]$. Studies have described two distinct clinical pictures based on Donahue et al.'s classification: Candida chorioretinitis or Candida endophthalmitis (when vitreous involvement occurs) [7]. These patients can be asymptomatic, or so systemically unwell that they are unable to communicate their visual symptoms. For this reason, the Infectious Diseases Society of America (IDSA) recommends fundoscopy screening for all fungal blood culture positive patients [8]. In contrast, the European Society of Clinical Microbiology and Infectious Diseases' (ESCMID) publication regarding the Diagnosis and Management of Candida Diseases does not even mention the potential for ocular involvement [9].

The Royal College of Ophthalmologists (RCOphth) recently published guidance in collaboration with the Intensive Care Society, stating that intensive care unit (ICU) 
patients are more likely to be non-verbal, therefore fundoscopy screening should be performed on all ICU patients with positive-fungal cultures [10]. In our health board, NHS Greater Glasgow and Clyde (NHSGGC), the local guidelines recommend fundoscopy screening should be performed on all patients after any positive-candida blood culture result. This screening recommendation is largely based on historical papers with higher prevalence rates, and from the USA population, where disseminated candidaemia is more common [11]. Considering this, we wished to audit the impact of the current guidelines on our department, and so determine if routine screening by ophthalmology within NHSGGC is warranted.

\section{Methods}

This retrospective observational study was performed in NHSGGC, which serves a population of 1.15 million in the West of Scotland. After liaison with our local microbiology service, a list of all patients with candida positive blood culture results over a 2-year period was obtained. The regional laboratory receives samples from many hospitals within the area, including two large teaching hospitals, several smaller District General and Ambulatory Care Hospitals and the regional cancer centre. As most of the published literature focused on adults, patients under the age of 17 were excluded and repeated cultures with the same species were counted as one. A retrospective case notes review was performed on all identified patients. Data pertaining to patients' demographics, candida species, antifungal therapy, patients' verbal status, presence of ocular symptoms, referral to ophthalmology and ophthalmic examination findings were collated.

\section{Results}

Between January 1, 2015 and December 31, 2016, 258 candida positive cultures were obtained from 168 adults over the age of 17 within NHSGGC. The mean age of the patients was 62 years (range: 17-94 years) with a gender ratio of 1:1 (85 females, 83 males). As expected, the highest number of positive cultures $(73.8 \%)$ were collected from patients admitted to the two largest university teaching hospitals in Glasgow (Glasgow Royal Infirmary and Queen Elizabeth University Hospital). Candida species were isolated in 161/168 (95.8\%) cases, with the remaining cultures growing yeasts and other fungal isolates (See Table 1). Candida albicans accounted for $43.5 \%$ of the positive cultures (70/168), closely followed by Candida glabrata (60/168, 35.7\%). All 168 patients received intravenous antifungal therapy shortly after the positive culture result,
Table 1 Fungal species isolated from blood cultures in NHS Greater Glasgow and Clyde 2015-2016

\begin{tabular}{lll}
\hline Species & Number & Percentage \\
\hline Candida albicans & 70 & 43.5 \\
Candida glabrata & 60 & 35.7 \\
Candida parapsilosis & 13 & 7.7 \\
Candida tropicalis & 6 & 3.6 \\
Candida dubliniensis & 5 & 3.0 \\
Candida lusitaniae & 3 & 1.8 \\
Candida guilliermondi & 3 & 1.8 \\
Yeast spp & 3 & 1.8 \\
Malassezia furfur & 2 & 1.2 \\
Cryptococcus neoformans & 1 & 0.6 \\
Trichosporon asteroides & 1 & 0.6 \\
Saccharomyces cerevisiae & 1 & 0.6 \\
Total & $\mathbf{1 6 8}$ & $\mathbf{1 0 0}$ \\
\hline
\end{tabular}

Table 2 Systemic antifungal therapy used in 84 fungal blood culture positive patients referred to ophthalmology

\begin{tabular}{lll}
\hline Systemic antifungal therapy & Number & Percentage \\
\hline Fluconazole & 41 & 48.8 \\
Micafungin & 19 & 22.6 \\
Caspofungin & 15 & 17.9 \\
Amphotericin & 8 & 9.5 \\
Andilafungin & 1 & 1.2 \\
Total & $\mathbf{8 4}$ & $\mathbf{1 0 0}$ \\
\hline
\end{tabular}

and therefore were on systemic treatment at the time of referral (See Table 2).

Of the 168 patients with positive-fungal culture results, only $84(50 \%)$ were formally referred to ophthalmology for fundoscopy. Of the patients that were not referred, 21 were deceased prior to the culture result $(21 / 168,12.5 \%), 14 \mathrm{had}$ subsequently deteriorated or deceased shortly after the culture results $(14 / 168,8.3 \%)$ or had fundoscopy performed by the ward doctor looking after them $(3 / 168,1.8 \%)$. In 46 patients $(27.4 \%)$ there was no recorded documentation of either fundoscopy or an ophthalmology referral.

Of the 84 patients referred to ophthalmology, $80(80 / 84$, $95.2 \%)$ underwent dilated fundoscopy. Over half of these patients $(49 / 80,61.3 \%)$ were able to communicate their visual symptoms and able to attend for evaluation in an outpatient setting, while the remainder were reviewed at the bedside. Six patients $(6 / 80,7.5 \%)$ had reported visual symptoms; five with blurred vision, and one with floaters. Almost two-thirds of patients who underwent fundoscopy had no ocular clinical findings $(52 / 80,65 \%)$, and $32.5 \%$ (26/80) had unrelated pre-existing ocular signs such as diabetic eye disease, macular degeneration, cataracts and 
Table 3 Summary of reported prevalence of intraocular candidiasis in patients with disseminated candidaemia

\begin{tabular}{llll}
\hline Author & Years of study & No. of patients examined & Total prevalence (\%) \\
\hline Donahue et al. & 1994 & 118 & 9.3 \\
Rodriguez-Adrian et al. & 1993-1994 & 180 & 5.0 \\
Scherer et al. & Jan 94-Apr 96 & 107 & 2.8 \\
Feman et al. & Feb 95-Aug 00 & 82 & 2.4 \\
Krishna et al. & May 96-Mar 97 & 31 & 26 \\
Oude Lashof et al. & 1998-2003 & 370 & 10.8 \\
Khalid et al. & Feb 00-Mar 10 & 144 & 12.5 \\
Geraymoych et al. & Jan 00-Dec 07 & 132 & 6.9 \\
Popovich et al. & 2003-2005 & 80 & 6.0 \\
Adam et al. & Jan 06-Dec 12 & 227 & 4.8 \\
Shah et al. & Jun 06-Nov 06 & 38 & 7.9 \\
Dozier et al. & Jun 06-Sep 09 & 211 & $<1$ \\
Ghodasra et al. & Jan 08-Dec 12 & 238 & 9.2 \\
Huynh et al. & Oct 09-Jun 11 & 49 & 0.5 \\
El-Abiary et al. (this study) & Jan 15-Dec 16 & $\mathbf{8 0}$ & $\mathbf{1 . 3}$ \\
\hline
\end{tabular}

optic neuropathy. One patient was examined by an ophthalmologist, but there was no specific documentation relating to this review identified in the patient's casenotes.

Only one patient had ocular signs consistent with candida chorioretinitis, making a prevalence of intraocular candida in our population of $1.3 \%$ (1/80). This patient was already on intravenous fluconazole at the time of referral, and unfortunately, after discharge, did not attend ophthalmic follow up. There were no documented cases of candida endophthalmitis identified in this cohort of 80 patients.

\section{Discussion}

Historically, the reported rates of intraocular candida infection have been as high as $28-45 \%$ for patients with disseminated candidaemia $[5,6,12]$. However, these studies included cotton wool spots and Roth Spots, signs which are not specific or diagnostic for ocular candidiasis, and have most likely resulted in an overestimation of the prevalence. Recently, published literature has generally used the classification of Donahue et al. [7], which described specific findings of either candida chorioretinitis or candida endophthalmitis. Reported prevalence based on these findings alone ranged from 2.4 to $25.8 \%$ up to end of the $1990 \mathrm{~s}$, then fell to $0.9-7.9 \%$ in the following decade (see Table 3) [13-16]. Our study revealed one possible case of intraocular candida infection from 80 patients over a 2-year period, making the presumed prevalence of $1.3 \%$ within Glasgow for 2015-2016. Although, this is consistent with the low-rates published in the literature, it is likely to be an underestimation, as only $50 \%$ (84/168) of the fungal culture positive patients were referred to ophthalmology in our series.
There are three suggested reasons in the published literature for this decrease in ocular candidiasis. Firstly, there is a higher suspicion for candidaemia in septicaemic patients, and with diagnostic tests more readily available, patients receive targeted therapy earlier. Newer generation antifungal agents, when administered systemically, have good intraocular bioavailability and more favourable clinical outcomes, particularly when treatment is commenced at an early stage [17]. The second reason could be low-capture rates. Patients with candidaemia can become critically ill, with attributable mortality rates ranging from 14 to $49 \%$, meaning a formal referral to ophthalmology for fundoscopy is not deemed an urgent clinical priority [18]. This was reflected in our dataset, with 35 out of the 168 patients (21\%) not being referred either due to deterioration in their general state of health, or mortality before or shortly after the culture result was obtained. Accurately identifying the true prevalence of ocular candidiasis would require a prospective approach, with dilated fundoscopy being performed on all fungal culture positive patients irrespective of their general health. The third reported reason is that it is often the less-experienced junior ophthalmologists who review such patients [19]. In our study, $18 \%$ of ophthalmic examinations were performed by a first- or secondyear trainee at the bedside with binocular indirect ophthalmoscopy.

Some studies have reported that patients can develop retinal lesions consistent with intraocular candida even after the initial negative fundoscopic screen [5, 6, 14, 19]. Despite these numbers being small, some authors conclude that repeat fundoscopic screening is warranted 2 weeks later, regardless if patients are asymptomatic and/or on appropriate systemic treatment. This recommendation is 
echoed in the IDSA guidelines. This scenario was not documented to have occurred in any of our cohort.

The impact of the presence or absence of visual symptoms remains controversial as to whether routine screening should be implemented. In a study by Adam et al., where 11 out of 227 patients were found to have intraocular involvement, 7 of them were able to verbalise ( 2 were asymptomatic and 5 complained of visual disturbances; positive predictive value $23.8 \%$ ) and 4 were non-verbal [20]. They calculated a sensitivity of $71.4 \%$ and a specificity of $89.3 \%$ with regards to visual symptoms as a predictor of ocular involvement, and advocated the importance of screening. In 2014, a review of 238 patients with a prevalence of ocular candida infection of $9.2 \%(22 / 238)$ found a specificity of $92.8 \%$ and a negative predictive value of $93.4 \%$ when predicting ocular disease [21]. However, more recently, Paulus et al. [22] published a large prospective study of fungal chorioretinitis and endophthalmitis and suggested the presence of symptoms should not be used as a screening tool, given the poor correlation with intraocular candidiasis. In contrast to this, only small numbers of patients with confirmed fundoscopic signs report visual symptoms. Dozier et al. evaluated 211 patients with an ocular candidiasis rate of $0.95 \%(2 / 211)$, and found that no asymptomatic patients had fundoscopic signs. In our study, the six patients who reported visual symptoms did not have ocular signs consistent with candidiasis. The one patient with confirmed fundoscopic signs was asymptomatic.

The management of ocular candidiasis is not standardised. Conventional treatments such as amphotericin B, and echinocandins such as caspofungin, have limited ocular penetration [23]. There is convincing evidence that the azole group of antifungals, such as fluconazole, have very good ocular penetration, and are therefore the preferred option when intraocular signs are confirmed [17, 24-26]. Current clinical practice suggests that systemic therapy is indicated in cases of chorioretinitis, and intravitreal injection of an antifungal agent is reserved for those patients with vitreous involvement. Intravitreal voriconazole is increasingly used to successfully manage candida endophthalmitis, but it is not without risks [27-30]. Vitrectomy is indicated when the vitreous is significantly affected, and has shown favourable outcomes when combined with an intravitreal injection [31-34]. We are unaware of any study to date comparing the outcomes of oral versus intravitreal voriconazole in this patient group.

There is little evidence in the literature to suggest that identifying ocular candidiasis through screening results in a change of management. The IDSA guidelines states that ocular signs dictate the mode and duration of antifungal treatment [8]. However, in 1994, Donahue et al. [7] observed that candida chorioretinitis did not progress to endophthalmitis when patients were on systemic antifungal therapy, hypothesising that systemic treatment was effective in preventing progression. More recently, a large prospective study in 2011 evaluating 370 patients with disseminated candidaemia mirrored this conclusion [35]. Their chorioretinitis and endophthalmitis rates were $9.2 \%$ and $1.6 \%$, respectively. Again, they noted that none of the chorioretinitis cases progressed to endophthalmitis, while on systemic antifungal therapy, and none required intravitreal injections. In our study, all patients were already being treated with systemic antifungal therapy at time of referral to ophthalmology, and management did not change in our single case.

The financial implications of recommending universal fundoscopic screening in cases of fungal positive blood culture patients is not discussed in the previously mentioned guidelines. There appears to be only one publication which involves a cost analysis, and this is based in the USA [21]. Ghodasra et al. found that on average, 26.4 inpatients had to be examined to find one patient who required a change in management. Incorporating the cost of new and return consultations, the cost of finding a single patient whose management subsequently changed was $\$ 50,582.98$ and was deemed to not be cost-effective.

\section{Conclusion}

With azole antifungal agents that have good intraocular penetration, ocular candidiasis rates appear to be decreasing. Within NHS GGC, all candida blood culture positive patients were receiving systemic antifungal therapy as per local protocol at the time of referral, the reported prevalence is low, and management rarely changes if ocular disease is found. Our findings support the view that routine fundoscopy screening is not indicated in every culture positive patient and provide an evidence base for the Royal College's recommendations for Eyecare in ICU, that being targeted screening of patients who are non-verbal, symptomatic, or high risk.

\section{Summary}

\section{What was known before}

- Worldwide prevalence of disseminated candidaemia is rising.

- Reported intraocular candidiasis rates are variable, even as low as $1 \%$.

- The Infectious Diseases Society of America recommends fundoscopy screening for all fungal blood culture positive patients. 
- The RCOphth have recently issued guidelines regarding eye care within the Intensive Care setting

\section{What this study adds}

- We report a low prevalence of ocular candidiasis (1.3\%) in our 2-year study.

- Our findings support the emerging view that routine fundoscopy screening may not be indicated in every culture positive patient.

- Targeted screening of patients who are non-verbal, symptomatic, or have risk factors for developing intraocular candidiasis may be more appropriate.

- Our study provides an evidence base for the RCOphth guidelines for eye care in the ICU.

Acknowledgements A version of this study was an oral presentation at the Scottish Ophthalmological Club meeting in February 2018, and at the Oxford Ophthalmological Congress in July 2018.

\section{Compliance with ethical standards}

Conflict of interest The authors declare that they have no conflict of interest.

\section{References}

1. Pfaller MA, Diekema DJ. Epidemiology of invasive candidiasis: a persistent public health problem. Clin Microbiol Rev. 2007;20:133-63.

2. Odds FC, Hanson MF, Davidson AD, Jacobson MD. One year prospective survey of Candida bloodstream infections in Scotland. J Med Microbiol. 2007;56:1066-75.

3. Vazquez JA, Sobel JD: Candidiasis. In: Clinical Mycology. Dismukes WE, Pappas PG, Sobel JD. Oxford University Press, Oxford, UK; 2003. p. 143-187.

4. Yapar N. Epidemiology and risk factors for invasive candidiasis. Ther Clin Risk Manag. 2014;10:95-105.

5. Brooks R. Prospective study of candida endophthalmitis in hospitalised patients with candidaemia. Arch Intern Med. 1989; 149:2226-8.

6. Parke DW, Jones DB, Gentry LO. Endogenous endophthalmitis among patients with candidaemia. Ophthalmology. 1982;89:789-96.

7. Donahue SP, Greven CM, Zuravleff JJ, Eller AW, Nguyen MH, Peacock JE Jr et al. Intraocular candidiasis in patients with candidemia: clinical implications derived from a prospective multicenter study. Ophthalmology . 1994;101:1302-1209.

8. Pappas PG, Kauffman CA, Andes DR, Clancy CJ, Marr KA, et al. Infectious Diseases Scoety of America: clinical and practical guidelines for the management of candidiasis. Clin Inf Dis. 2016;62:1-50.

9. Fungal Infection Study Group. ESCMID Guideline for the Diagnosis and Management of Candida Diseases 2012. European Society of Clinical Microbiology and Infectious Diseases; 2012.

10. Lightman SMH Eye Care in ICU. Clinical Guidance. Royal College of Ophthalmologists and Intensive Care Society; 2017.

11. Vinikoor MJ, Zoghby J, Cohen KL, Tucker JD. Do all candidaemic patients need an ophthalmic examination? Int J Infect Dis. 2014;17:146-8.
12. Edwards JE Jr, Foos RY, Montgomerie JZ, Guze LB. Ocular manifestations of candida septicaemia: review of 76 cases of haemtogenous candida endophthalmitis. Medicine. 1974; 53:47-75.

13. Feman SS, Nichols JC, Chung SM, Theobald TA. Endopthalmitis in patients with disseminated fungal disease. Trans Am Ophthalmol Soc. 2002;100:67-72.

14. Krishna R, Amuh D, Lowder CY, Gordon AM, Adal KA, Hall G. Should all patients with candidaemia have an ophthalmic examination to rule out ocular candidiasis? Eye. 2000;14:30-34.

15. Dozier CC, Tarantola RM, Jiramongkolchai K, Donahue SP. Fungal eye disease at a tertiary care centre: the utility of routine inpatient consultation. Ophthalmology. 2011;118:1671-6.

16. Shah CP, McKey J, Spirn MJ, Maguire J. Ocular Candidiasis: a review. Br J Ophthalmol. 2008;92:466-8.

17. Khan FA, Slain D, Khakoo RA. Candida endophthalmitis: focus on current and future antifungal treatment options. Pharmacotherapy. 2007;27:1711-21.

18. Zaoutis TE, Argon J, Chu J, Berlin J, Walsh T, Feudtner C. The epidemiology and attributable outcomes of candidemia in adults and children hospitalized in the United States: a propensity analysis. Clin Infect Dis. 2005;41:1232-9.

19. Geramovych E,Conduff JH,Barich PS,Leffler CT,Brar VS, Prevalence and factors predictive of intraocular fungal infection in patients with fungaemia at an Urban Tertiary Care Center. Clin Ophthalmol. 2015;9:1853-8.

20. Adam MK, Vahedi S, Nichols MM, Fintelmann RE, Keenan JD, Garg SJ et al. Inpatient ophthalmology consultation for fungaemia: prevalence of ocular involvement and necessity of fundoscopic screening. Am J Ophthal. 2015; 160:1078-83.

21. Ghodasra DH, Eftekhari K, Shah AR, Vanderbeek. Outcomes, impact on management, and costs of fungal eye disease consults in a tertiary care setting. Ophthalmology. 2014;121:2334-8.

22. Paulus YM, Cheng S, Karth PA, Leng T. Prospective trial of endogenous endophthalmitis and chorioretinitis rates, clinical course and outcomes in patients with fungaemia. Retina. 2016;36:1357-63.

23. Felton T, Troke PF, Hope WW. tissue penetration of antifungal agents. Clin Microbiol Rev. 2014;27:68-88.

24. Riddell J, Comer GM, Kauffmann CA. Treatment of endogenous fungal endophthalmitis: focus on new antifungal agents. Clin Infect Dis. 2011;52:648-53.

25. Savani DV, Perfect JR, Cobo LM, Durack DT. Penetration of new azole compounds into the eye and efficacy in experimental candida endophthalmitis. Antimicrob Agents Chemother. 1987; 31:6-10.

26. Hariprasad SM, Mieler WF, Holz ER. Determination of vitreous, aqueous, and plasma concentration of orally administered voriconazole in humans. Arch Ophthalmol. 2004;122:42-47.

27. Varma D, Thaker HR, Moss PJ, Wedgwood K, Innes JR. Use of voriconazole in Candida retinitis. Eye. 2005;19:485-7.

28. Hariprasad SM, Mieler WF, Lin TK, Sponsel WE, Graybill JR. Voriconazole in the treatment of fungal eye infections: a review of current literature. Br J Ophthalmol. 2008;92:871-8.

29. Jang GJ, Kim KS, Shin WS, Lee WK. Treatment of candida chorioretinitis with voriconazole. Korean J Ophthalmol. 2005; 19:73-76.

30. Breit SM, Hariprasad SM, Mieler WF, Shah GK, Mills MD, Grand MG. Management of endogenous fungal endophthalmitis with voriconazole and caspofungin. Am J Ophthalmol. 2005; 139:135-40.

31. Martinez-Vazquez C, Fernandez-Ulloa J, Bordon J. Candida albicans endophthalmitis in brown heroin addicts: response to early vitrectomy preceded and followed by antifungal therapy. Clin Infect Dis. 1998;27:1130-3. 
32. Zhang YQ, Wang WJ. Treatment outcomes after pars plana vitrectomy for endogenous endophthalmitis. Retina. 2005; 25:746-50.

33. Barrie T. The place of elective vitrectomy in the management of patients with candida endophthalmitis. Graefes Arch Clin Exp Ophthalmol. 1987;225:107-13.
34. Snip RC, Michels G. Pars plana vitrectomy in the management og endogenous endophthalmitis. Am J Ophthalmol. 1976 ;82:699-704.

35. Oude Lashof AML, Rothova A, Sobel J, Ruhnke M, Pappas PG, Viscoli $\mathrm{C}$ et al. Ocular manifestations of candidaemia. Clin Infect Dis. 2011;53:262-8. 